\title{
Hepatic failure induced by cyproterone acetate: A case report and literature review
}

\author{
Jae Heon Kim, MD, PhD; ${ }^{*}$ Byung Wook Yoo, MD, PhD; ${ }^{\dagger}$ Won Jae Yang, MD, PhD*
}

*Department of Urology, Soonchunhyang University Hospital, Soonchunhang University College of Medicine, Seoul, Korea; 'Department of Family Medicine, Soonchunhyang University Hospital, Soonchunhyang University College of Medicine, Seoul, Korea

Cite as: Can Urol Assoc J 2014;8(5-6):e458-61. http://dx.doi.org/10.5489/cuaj.1753 Published online June 19, 2014.

\section{Abstract}

Cyproterone acetate (CPA) is an anti-androgenic drug that has been used to manage prostate cancer. The drug is well-tolerated, but has hepatotoxic effects. Hepatic failure induced by CPA is rare urologists tend to overlook its severity. We report a patient with metastatic prostate cancer who developed CPA-induced hepatic failure that manifested as bilirubinuria, which was initially misinterpreted as gross hematuria. The patient died despite receiving critical care. The aim of this study is to sound the alarm about CPA-induced hepatic failure.

\section{Introduction}

Cyproterone acetate (CPA) is a steroidal synthetic progestagen and anti-androgenic compound widely administered in patients with metastatic prostate cancer. ${ }^{1}$ The first case of CPA-induced fulminant hepatitis with a fatal outcome was reported in $1989 .{ }^{2}$ A variety of hepatotoxic reactions have been documented, including immunoallergic cytotoxic reactions, ${ }^{3}$ cholestasis, autoimmune hepatitis, ${ }^{4}$ acute hepatitis, ${ }^{5}$ and fulminant hepatic failure. ${ }^{1-3,6-14}$ Despite its low incidence, the prognosis of hepatic failure induced by CPA is fatal. Only 1 of 14 reported patients has survived. . $^{-3,6-14}$

CPA has been widely prescribed as an anti-androgen to suppress the progression of metastatic prostate cancer. Considering the high use of CPA by urologists late into the treatment process, more discussion about the complication of this drug is needed. It is well-known that patients with prostate cancer have a relatively good prognosis and even patients with bone metastasis can have extended survival periods. ${ }^{15}$ Unfortunately, CPA-induced hepatic failure may encroach upon the considerably favourable survival period among patients with metastatic prostate cancer. We describe this rare phenomenon and review the relevant literature.

\section{Case report}

An 87-year-old male visited our urologic clinic due to acute urinary retention. He lived in the countryside and had never undergone any specific medical test. The patient complained of weak urinary stream, sense of incomplete voiding, hesitancy, straining to urinate, frequency, urgency, and nocturia. He also complained of pain around the pelvic and lumbar area. A digital rectal exam showed an enlarged prostate with multiple palpable nodules on both peripheral lobes. A Foley catheter was inserted through his urethra, and about $700 \mathrm{~mL}$ of urine was drained. Laboratory examination showed mild anemia, elevated serum prostate-specific antigen (PSA) $(>1000 \mathrm{ng} / \mathrm{mL}$ ) and elevated alkaline phosphatase. Liver enzyme and serum creatinine levels were within normal limits. Markers for viral hepatitis and autoimmune hepatitis were negative. Transrectal sonography demonstrated an enlarged prostate (about $70 \mathrm{~mL}$ in volume) with protrusion into the bladder neck. In light of the prostate cancer and the bladder outlet obstruction, we initiated palliative transurethral resection of prostate (TURP). Large kissing lobes were endoscopically resected. Histologic examination revealed prostatic adenocarcinoma, with Gleason sum $9(5+4)$. Both preoperative and postoperative aspartate transaminase (AST) and alanine transaminase (ALT), and bilirubin were within normal ranges. A whole body bone scan showed multiple hot uptake of radioisotope in the pelvic bone and lumbar spine, suggesting bony metastases (Fig. 1).

After he achieved successful self-voiding, he was discharged with daily $200 \mathrm{mg}$ of CPA and a gonadotropinreleasing hormone $(\mathrm{GnRH})$ agonist injection for maximal androgen blockade. Three months later, he called our institution and complained of intermittent dark pinkishcoloured urine, which was misinterpreted as a sustained mild gross hematuria following the TURP and was advised to drink plenty of water. The CPA medication was continued. Six months after the operation, he visited our clinic due to drowsy mental status and persistent dark pinkishcoloured urine (Fig. 2). On physical examination, the patient 


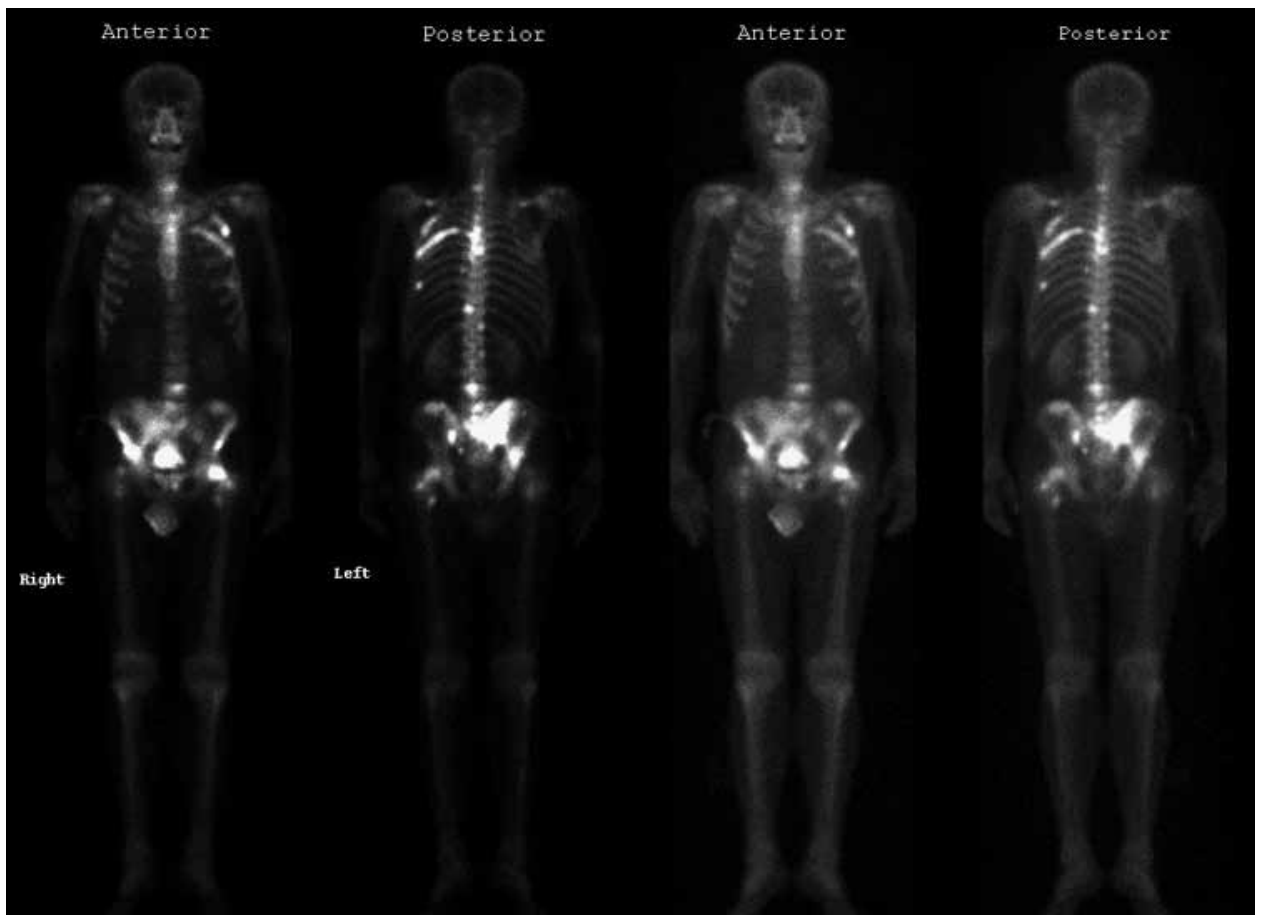

Fig. 1. Whole body bone scan.

was jaundice with a yellowish eye. His urinalysis revealed bilirubinuria with no red blood cell count on microscopic examination. His serum PSA had decreased to $174 \mathrm{ng} / \mathrm{mL}$. Laboratory test revealed mild anemia, elevated AST/ALT at $529 / 223 \mathrm{IU} / \mathrm{L}$, total and direct bilirubin at $10.6 \mathrm{mg} / \mathrm{dL}$ (range: $0.2-1.2$ ) and 5.2 (range: 0.0-0.4), respectively, ammonia at 294 (range: 25-65), lactic acid dehydrogenase at $452 \mathrm{U} / \mathrm{L}$ (range: 106-211), Gamma-glutamyl transferase (GGT) at $85 \mathrm{U} / \mathrm{L}$ (range: 11-49), and international normalized ratio (INR) 2.4, all of which suggested acute liver failure. Although CPA was discontinued immediately, the patient's condition continued to deteriorate with persistent elevation of total

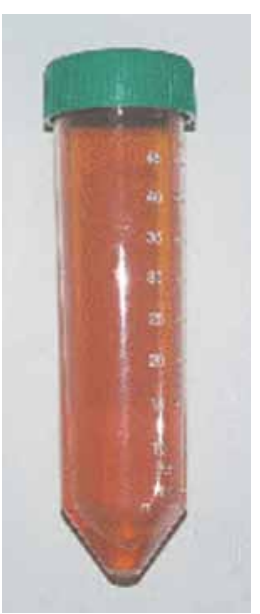

Fig. 2. Gross inspection of bilirubinuria. bilirubin level and he died 20 days after admission due to multi-organ failure.

\section{Discussion}

CPA is thought to be well-tolerated, but fulminant hepatic failures have been reported. The mechanism of CPAinduced hepatic toxicity is not wellknown. The histological features fit with an idiosyncratic reaction directly related to the drug or its metabolites, or possibly an immunologically mediated reaction. ${ }^{2,9} \mathrm{~A}$ retrospective study involving 2506 patients receiving CPA revealed that $9.6 \%$ of them eventually presented with pathological liver profile. ${ }^{16}$
Toxic hepatic failure in our patient appeared to be related to the administration of CPA based on the temporal relationship, negative serology for acute viral infection, negative autoantibody markers and exclusion of drugs or other potentially hepatotoxic agents. Hepatic failure is defined by the presence of encephalopathy and increased INR more than 1.5. Investigation via liver biopsy was not possible in many cases of CPA-induced hepatic failure due to ethical reasons.

In total, there are 15 reported cases of CPA-induced fulminant hepatic failure; all patients took prescription CPA due to prostate cancer. ${ }^{1-3,10-16}$ (Table 1). Adverse hepatic reactions occurred more commonly in elderly patients (age range: 65-92) with malignant diseases who were treated with higher doses (range: 100-300 mg) for a prolonged period.

Fulminant hepatic failure developed a few weeks to several months after initiation of therapy (range: 2-15 months). The biochemical profile showed that AST/ALT were 3 to 27 times higher than normal ranges and bilirubin were 9 to 30 times higher than normal. ${ }^{11}$ More prominent findings included elevated coagulation profiles, including INR and prothromin time. Among the cases, 6 patients $(40 \%)$ complained of dark urine. Dark-coloured urine and a history of treatment, such as radiation therapy, radical prostatectomy or TURP for prostate cancer, could be misdiagnosed as gross hematuria. Paradoxically, the known sole survivor also had alcoholic liver cirrhosis and the impending liver failure was detected early thanks to the close follow-up of his liver function. ${ }^{3}$ This implies an interesting and important message to urologists and other clinicians.

The 5-year survival rate of patients with metastatic prostate cancer is more than $50 \% .{ }^{15}$ Among the 15 reported cases of CPA-induced hepatic failure, 4 had bone metastasis and 3 were locally invasive or localized prostate cancer. No information on the specific stage of the prostate cancer was available in the other reported cases. Considering the fatal outcome of CPA-induced hepatic failure, urologists should be aware of this phenomenon. In patients taking CPA, regular follow-up of their hepatic function is warranted.

\section{Conclusion}

CPA-induced hepatic failure can encroach upon the overall survival period of patients with prostate cancer. Close 
Kim et al.

Table 1. CPA-induced hepatic failure: Review of 15 cases.

\begin{tabular}{|c|c|c|c|c|c|c|c|c|c|c|}
\hline Reference & Age & $\begin{array}{l}\text { History of LC } \\
\text { or hepatitis }\end{array}$ & $\begin{array}{l}\text { Dose } \\
\text { (mg) }\end{array}$ & Dark urine & AST/ALT (IU/L) & $\begin{array}{l}\text { GGT } \\
\text { (IU/L) }\end{array}$ & $\begin{array}{c}\text { Bilirubin } \\
\text { (total/ } \\
\text { direct, } g / \mathrm{L} \\
\text { or } \mu \mathrm{mol} / \mathrm{L} \text { ) }\end{array}$ & $\begin{array}{l}\text { Medication } \\
\text { period }\end{array}$ & INR or PT & Prognosis \\
\hline $\begin{array}{l}\text { Current } \\
\text { case }\end{array}$ & 87 & No & 200 & Yes & $529 / 223$ & 85 & $10.6 / 5.2$ & 6 & 2.4 & Expired \\
\hline $\begin{array}{l}\text { Savidou et } \\
\text { al. }{ }^{1}\end{array}$ & 83 & No & 300 & Yes & $721 / 283$ & & $10.1 / 9.2$ & 7 & 2.24 & Expired \\
\hline $\begin{array}{l}\text { Levesque } \\
\text { et al. }{ }^{2}\end{array}$ & 78 & No & 200 & Yes & $720 / 1015$ & & $\begin{array}{c}178 / \text { not } \\
\text { remarked }\end{array}$ & 6 & $\begin{array}{c}\text { PT } 47 \% \text { of } \\
\text { NL }\end{array}$ & Expired \\
\hline $\begin{array}{l}\text { Murphy et } \\
\text { al. }^{3}\end{array}$ & 73 & $\begin{array}{c}\text { Yes (alcoholic } \\
\text { LC) }\end{array}$ & 300 & $\begin{array}{l}\text { Not } \\
\text { remarked }\end{array}$ & $736 /$ & 634 & $\begin{array}{c}\text { 193/not } \\
\text { remarked }\end{array}$ & 4 & 1.8 & Alive \\
\hline $\begin{array}{l}\text { Castellani } \\
\text { et al. }{ }^{6}\end{array}$ & 78 & No & $\begin{array}{c}200- \\
300\end{array}$ & $\begin{array}{l}\text { Not } \\
\text { remarked }\end{array}$ & $\begin{array}{l}18 \text { times upper/ } \\
9 \text { times upper }\end{array}$ & $\begin{array}{l}3 \text { times } \\
\text { upper }\end{array}$ & $\begin{array}{c}429 / \text { not } \\
\text { remarked }\end{array}$ & 3 & $\begin{array}{c}\text { PT } 21 \% \text { of } \\
\text { NL }\end{array}$ & Expired \\
\hline $\begin{array}{l}\text { Antoni et } \\
\text { al. } 7\end{array}$ & 80 & No & 200 & $\begin{array}{l}\text { Not } \\
\text { remarked }\end{array}$ & $\begin{array}{l}25 \text { times upper/ } \\
20 \text { times upper }\end{array}$ & $\begin{array}{l}2 \text { times } \\
\text { upper }\end{array}$ & $\begin{array}{l}472 / \text { not } \\
\text { remarked }\end{array}$ & $\begin{array}{c}\text { not } \\
\text { remarked }\end{array}$ & & Expired \\
\hline Parys et al. ${ }^{8}$ & 65 & No & 300 & $\begin{array}{l}\text { Not } \\
\text { remarked }\end{array}$ & $377 / 252$ & 512 & $\begin{array}{c}177 / \text { not } \\
\text { remarked }\end{array}$ & 12 & & Expired \\
\hline Parys et al. ${ }^{8}$ & 83 & No & 300 & Yes & $155 / 79$ & 65 & $\begin{array}{l}281 / \text { not } \\
\text { remarked }\end{array}$ & 21 & & Expired \\
\hline $\begin{array}{l}\text { Friedmann } \\
\text { et al. }{ }^{9}\end{array}$ & 81 & No & 300 & Yes & $515 / 395$ & 455 & $\begin{array}{l}513 / \text { not } \\
\text { remarked }\end{array}$ & 6 & PT 16.5sec & Expired \\
\hline $\begin{array}{l}\text { Friedmann } \\
\text { et al. }{ }^{9}\end{array}$ & 66 & No & 300 & $\begin{array}{l}\text { Not } \\
\text { remarked }\end{array}$ & $946 / 702$ & 375 & $\begin{array}{l}\text { 208/not } \\
\text { remarked }\end{array}$ & 2 & 2.5 & Expired \\
\hline $\begin{array}{l}\text { Hirsch et } \\
\text { al. }{ }^{10}\end{array}$ & 92 & No & 100 & $\begin{array}{l}\text { Not } \\
\text { remarked }\end{array}$ & $1,020 / 1,80$ & 238 & $\begin{array}{l}20 / \text { not } \\
\text { remarked }\end{array}$ & 4 & $\begin{array}{c}\text { PT } 34 \% \text { of } \\
\text { NL }\end{array}$ & Expired \\
\hline $\begin{array}{l}\text { Lombardi } \\
\text { et al. }{ }^{11}\end{array}$ & 84 & No & 300 & $\begin{array}{l}\text { Not } \\
\text { remarked }\end{array}$ & $778 / 746$ & 191 & $505 / 270$ & & & Expired \\
\hline $\begin{array}{l}\text { Bressollete } \\
\text { et al. } .^{12}\end{array}$ & 79 & No & 300 & $\begin{array}{l}\text { Not } \\
\text { remarked }\end{array}$ & $\begin{array}{l}9 \text { times upper/ } \\
\text { not remarked }\end{array}$ & & $\begin{array}{l}30 \text { times } \\
\text { upper than } \\
\mathrm{NL} / \text { not } \\
\text { remarked }\end{array}$ & 10 & $\begin{array}{c}\text { PT } 18 \% \text { of } \\
N L\end{array}$ & Expired \\
\hline Hsu et al. ${ }^{13}$ & 71 & No & 100 & Yes & $245 / 255$ & & $8.2 / 4.5$ & 4 & PT $15.2 \mathrm{sec}$ & Expired \\
\hline Kim et al. ${ }^{14}$ & 89 & No & 300 & $\begin{array}{l}\text { Not } \\
\text { remarked }\end{array}$ & $1,008 / 1,089$ & 191 & $\begin{array}{l}23 / \text { not } \\
\text { remarked }\end{array}$ & 3 & $\begin{array}{c}1.9 \\
\text { PT } 59.6 \mathrm{sec}\end{array}$ & Expired \\
\hline
\end{tabular}

LC: liver cirrhosis; AST/ALT: aspartate transaminase/alanine transaminase; GGT: gamma-glutamyl transferase; INR: international normalized ratio; PT: prothromin time; NL: normal.

monitoring of liver function is recommended to prevent this fatal complication.

Competıng interests: Dr. Kim, Dr. Yoo and Dr. Yang all declare no competing financial or personal interests.

This paper has been peer-reviewed.

Acknowledgement: This work was partially supported by Soonchunhyang University Research Fund.

\section{References}

1. Savidou I, Deutsch M, Soultati AS, et al. Hepatotoxicity induced by cyproterone acetate: a report of three cases. World J Gastroenterol 2006:12:7551-5.
2. Levesque $\mathrm{H}$, Trivalle $\mathrm{C}$, Manchon ND, et al. Fulminant hepatitis due to cyproterone acetate. Lancet 1989:1:215-6. http://dx.doi.org/10.1016/S0140-6736(89)91225-7

3. Murphy BJ, Collins BJ. Severe hepatitis and liver failure induced by cyproterone acetate. Aust $N Z$ I J Med 1996:26:724. http://dx.doi.org/10.1111/i.1445-5994.1996.tb02956.x

4. Kacar $S$, Akdogan M, Kosar $Y$, et al. Estrogen and cyproterone acetate combinationinduced autoimmune hepatitis. J Clin Gastroenterol 2002:35:98-100. http://dx.doi.org/10.1097/00004836-20020700000023

5. Drakos PE, Gez E, Catane R. Hepatitis due to cyproterone acetate. Eur J Cancer 1992;28A:1931-2. http://dx.doi.org/10.1016/0959-8049(92)90041-Y

6. Castellani P, Bernardini D, Renou C, et al. Fatal sub-fulminant hepatitis caused by cyproterone acetate. A new case. Gastroenterol Clin Biol 1996:20:915-6.

7. Antoni $M$, Bourliere $M$, Toullec J, et al. Fatal subfulminant hepatitis caused by cyproterone acetate. Gastroenterol Clin Biol 1991:15:772-3.

8. Parys BT, Hamid S, Thomson RG. Severe hepatocellular dysfunction following cyproterone acetate therapy. BrJ Urol 1991:67:312-3. http://dx.doi.org/10.1111/i.1464-410X.1991.tb15142.x

9. Friedman $G$, Lamoureux $E$, Sherker AH. Fatal fulminant hepatic failure due to cyproterone acetate. Dig Dis Sci 1999:44:1362-3. http://dx.doi.org/10.1023/A:1026639432428

10. Hirsch D, Kovatz S, Bernheim J, et al. Fatal fulminant hepatitis from cyproterone acetate. Isr J Med Sci 1994:30:238-40.

11. Lombardi A, Ferrazza P, Castaldi F, et al. Acute hepatic necrosis in a patient treated with cyproterone acetate. GChir 1998:19:161-3. 
12. Bressollette L, Dubois A, Carhant D, et al. Fatal hepatitis caused by cyproterone acetate. Therapie 1994:49:153.

13. Hsu YC, Tai DI. Unusually high alanine aminotransferase to aspartate aminotransferase ratio in a patient with cyproterone-induced icteric hepatitis. Chang Gung Med J 2011:34(6 Suppl):34-8.

14. Kim BH, Kim DJ, Sohn KM, et al. A case of fulminant hepatic failure due to cyproterone acetate in a patient with cryptogenic liver cirrhosis. Korean J Med 2009:77:S31-5.

15. Birtle A. Maximizing survival in metastatic castrate-resistant prostate cancer: A clinical viewpoint. Expert Rev Anticancer Ther 2013:13:89-99. http://dx.doi.org/10.1586/era.12.160
16. Rabe T, Feldmann K, Heinemann L, et al. Cyproterone acetate: is it hepato- or genotoxic? Drug Saf 1996:14:25-38. http://dx.doi.org/10.2165/00002018-199614010-00004

Correspondence: Dr. Won Jae Yang, Department of Urology, Soonchunhyang University Hospital, Soonchunhang University College of Medicine, Seoul, Korea; woniya@schmc.ac.kr 